\title{
DYNAMIC ANALYSIS ON THE SINGLE LINE QUEUEING MODEL WITH REPEATED DEMANDS
}

\author{
EHMET KASIM*, GENI GUPUR
}

College of Mathematics and System Sciences, Xinjiang University, Urumqi 830046, China

\begin{abstract}
In this paper, we consider the single line queueing model with repeated demands which is described by infinitely many partial differential equations with boundary conditions. By using the strong continuous semigroup theory of linear operators, we prove the existence of the unique positive time-dependent solution of the model. We also investigate the asymptotic behavior of the time-dependent solution and deduce that the time-dependent solution of the model strongly converges to its steady-state solution.
\end{abstract}

Keywords. Single line queueing model; $C_{0}$-semigroup; Dispersive operator; Resolvent set; Eigenvalue.

2010 Mathematics Subject Classification. 47D03, 47A10, 60K25.

\section{INTRODUCTION}

From Farahmand [1], the single-line queueing model with repeated demands can be described by the following partial differential equations:

$$
\begin{aligned}
& \frac{d p_{0}(t)}{d t}=-\lambda p_{0}(t)+\int_{0}^{\infty} \eta(x) W_{0}(x, t) d x, \\
& \frac{d p_{n}(t)}{d t}=-(\lambda+\xi) p_{n}(t)+\int_{0}^{\infty} \eta(x) W_{n}(x, t) d x, \quad n \geq 1, \\
& \frac{\partial W_{0}(x, t)}{\partial t}+\frac{\partial W_{0}(x, t)}{\partial x}=-(\lambda+\eta(x)) W_{0}(x, t), \\
& \frac{\partial W_{n}(x, t)}{\partial t}+\frac{\partial W_{n}(x, t)}{\partial x}=-(\lambda+\eta(x)) W_{n}(x, t)+\lambda W_{n-1}(x, t), \quad n \geq 1, \\
& W_{n}(0, t)=\lambda p_{n}+\xi p_{n+1}, \quad n \geq 1, \quad p_{m}(0)=0, \quad m \geq 1, \quad W_{n}(x, 0)=0, \quad n \geq 0, \\
& p_{0}(0)=1, \quad p,
\end{aligned}
$$

${ }^{*}$ Corresponding author.

E-mail addresses: ehmetkasim@163.com (K. Ehmet), geni@xju.edu.cn (G. Gupur).

Received April 8, 2019; Accepted November 25, 2019.

(C)2019 Journal of Nonlinear Functional Analysis 
where $(x, t) \in[0, \infty) \times[0, \infty), p_{n}(t)$ denotes the probability that the server is idle and $n$ customers are in orbit, $W_{n}(x, t)$ stands for the joint probability that at time $t$ there are $n$ customers in orbit and a customer in service with elapsed service time lying in $(x, x+d x], \lambda$ is the mean arrival rate of customers, $\xi$ is the repeated rate of customers in the orbit, $\eta(x)$ is the service completion rate at time $x$ satisfying

$$
\eta(x) \geq 0, \quad \int_{0}^{\infty} \eta(x) d x=\infty
$$

Retrial queueing (or queueing with repeated demands) systems have been widely used to model many problems in telephone switching systems, computer and communication systems. Many researchers studied such queueing system; see, e.g., Falin and Templeton [2], Atencia et al. [3], Artalejo and GomezCorral [4], Choi, Melikov and Velibekov [5], Choudhury [6], Wang, Zhao and Zhang [7] for the steadystate analysis, and Gupur [8, 9], Kasim and Gupur [10], Gao and Zhu [11] for the dynamical analysis.

Retrial queues have a significant difference with the usual queueing systems that, with retrial queues, the server cannot be in continuous contact with the waiting customers, who can only call in to test the state of the server. If the server is free, service commences immediately. However, if the server is occupied, the customer must break contact and reinitiate their request later. These unsatisfied customers produce a source of customers called the orbit. Therefore, the server receives requests from arrivals, from outside at a rate $\lambda$, and from customers in the orbit at a rate $\xi_{n}$ when the orbit size is $n$ according to the Poisson process. Farahmand [1] considered the case of discouraged repeated demands, $\xi_{n}=\xi / n$, in which the customers from orbit are relaxed, and are prepared to reduce their rate of service as more customers join the orbit. Obviously, the competition to find the server idle is higher. Farahmand established the above model by using the supplementary variable technique and gave the several characteristic quantities of this queueing model in steady-state case under the hypothesis that "the time-dependent solution of the model converges to a nonzero steady-state solution". However, Farahmand did not answer if the above hypothesis holds. We know that it is not always hold [9]. Therefore, it needs to study above hypothesis. This paper is an effort on this subject. We investigate dynamic analysis for above queueing model. First, we transform the model into an abstract Cauchy problem, and, by using the $C_{0}$ - semigroup theory, prove that the model has a unique positive time-dependent solution which satisfies the probability condition. Next, by studying spectral properties of the underlying operator, which corresponds to the model, we obtain that the time-dependent solution converges to the steady-state solution.

Take a state space as follows.

$$
X=\left\{\begin{array}{l|l}
(p, W) & \begin{array}{l}
p_{0} \in l^{1}, W \in Y \\
\|(p, W)\|=\|p\|_{l^{1}}+\|W\|_{Y}<\infty
\end{array}
\end{array}\right\}
$$

where

$$
Y=\left\{\begin{array}{l|l}
W & \begin{array}{l}
W=\left(W_{0}, W_{1}, W_{2}, \cdots\right) \in L^{1}[0, \infty) \times L^{1}[0, \infty) \times L^{1}[0, \infty) \times \cdots, \\
\|W\|=\sum_{n=0}^{\infty}\left\|W_{n}\right\|_{L^{1}[0, \infty)}
\end{array}
\end{array}\right\}
$$


It is obvious that $X$ is a Banach space. We now define a maximal operator $\left(A_{m}, D\left(A_{m}\right)\right)$ and its domain as:

$$
\begin{aligned}
& A_{m}\left(\left(\begin{array}{c}
p_{0} \\
p_{1} \\
p_{2} \\
p_{3} \\
\vdots
\end{array}\right),\left(\begin{array}{c}
W_{0}(x) \\
W_{1}(x) \\
W_{2}(x) \\
W_{3}(x) \\
\vdots
\end{array}\right)\right)=\left(\left(\begin{array}{cccc}
-\lambda & 0 & 0 & \ldots \\
0 & -(\lambda+\xi) & 0 & \ldots \\
0 & 0 & -(\lambda+\xi) & \ldots \\
\vdots & \vdots & \vdots & \ddots
\end{array}\right)\left(\begin{array}{c}
p_{0} \\
p_{1} \\
p_{2} \\
p_{3} \\
\vdots
\end{array}\right)\right. \\
& \left.+\left(\begin{array}{cccc}
\psi & 0 & 0 & \ldots \\
0 & \psi & 0 & \ldots \\
0 & 0 & \psi & \ldots \\
\vdots & \vdots & \vdots & \ddots
\end{array}\right)\left(\begin{array}{c}
W_{0}(x) \\
W_{1}(x) \\
W_{2}(x) \\
\vdots
\end{array}\right),\left(\begin{array}{ccccc}
\phi & 0 & 0 & 0 & \ldots \\
\lambda & \phi & 0 & 0 & \ldots \\
0 & \lambda & \phi & 0 & \ldots \\
0 & 0 & \lambda & \phi & \ldots \\
\vdots & \vdots & \vdots & \vdots & \ddots
\end{array}\right)\left(\begin{array}{c}
W_{0}(x) \\
W_{1}(x) \\
W_{2}(x) \\
W_{3}(x) \\
\vdots
\end{array}\right)\right), \\
& D\left(A_{m}\right)=\left\{\begin{array}{l|l}
(p, W) \in X & \begin{array}{l}
\frac{d W_{n}}{d x} \in L^{1}[0, \infty), W_{n}(x) \text { is absolutely } \\
\text { continuous and } \sum_{n=0}^{\infty}\left\|\frac{d W_{n}}{d x}\right\|_{L^{1}[0, \infty)}<\infty,
\end{array}
\end{array}\right\},
\end{aligned}
$$

where

$$
\begin{aligned}
\psi f & =\int_{0}^{\infty} \eta(x) f(x) d x, \quad f \in L^{1}[0, \infty), \\
\phi g & =-\frac{d g(x)}{d x}-(\lambda+\eta(x)) g(x), \quad g \in W^{1,1}[0, \infty) .
\end{aligned}
$$

We denote the boundary space of $X$ by

$$
\partial X=l^{1}
$$

and define two boundary operators by

$$
\begin{gathered}
L: D\left(A_{m}\right) \rightarrow \partial X, \quad \Phi: D\left(A_{m}\right) \rightarrow \partial X \\
L\left(\left(\begin{array}{c}
p_{0} \\
p_{1} \\
p_{2} \\
p_{3} \\
\vdots
\end{array}\right),\left(\begin{array}{c}
W_{0}(x) \\
W_{1}(x) \\
W_{2}(x) \\
W_{3}(x) \\
\vdots
\end{array}\right)\right)=\left(\begin{array}{c}
W_{0}(0) \\
W_{1}(0) \\
W_{2}(0) \\
W_{3}(0) \\
\vdots
\end{array}\right), \\
\Phi\left(\left(\begin{array}{c}
p_{0} \\
p_{1} \\
p_{2} \\
p_{3} \\
\vdots
\end{array}\right),\left(\begin{array}{c}
W_{0}(x) \\
W_{1}(x) \\
W_{2}(x) \\
W_{3}(x) \\
\vdots
\end{array}\right)\right)=\left(\begin{array}{cccccc}
\lambda & \xi & 0 & 0 & 0 & \ldots \\
0 & \lambda & \xi & 0 & 0 & \ldots \\
0 & 0 & \lambda & \xi & 0 & \ldots \\
0 & 0 & 0 & \lambda & \xi & \ldots \\
\vdots & \vdots & \vdots & \vdots & \vdots & \ddots
\end{array}\right)\left(\begin{array}{c}
p_{0} \\
p_{1} \\
p_{2} \\
p_{3} \\
\vdots
\end{array}\right) .
\end{gathered}
$$

If we define the operator $\mathscr{A}$ and its domain as

$$
\begin{aligned}
\mathscr{A}(p, W) & =A_{m}(p, W), \\
D(\mathscr{A}) & =\left\{(p, W) \in D\left(A_{m}\right) \mid L(p, W)=\Phi(p, W)\right\},
\end{aligned}
$$


then system (1.1)-(1.6) can be written as the following abstract Cauchy problem in Banach space $X$ :

$$
\left\{\begin{array}{l}
\frac{d(p, W)(t)}{d t}=\mathscr{A}(p, W)(t), \\
\left.(p, W)(0)=\left(\begin{array}{l}
1 \\
0 \\
\vdots
\end{array}\right),\left(\begin{array}{c}
0 \\
0 \\
\vdots
\end{array}\right)\right)
\end{array} \quad t \in(0, \infty)\right.
$$

\section{The Well-POSEDNES OF SYSTEM (1.7)}

In this section, we first prove that the operator generates a positive contraction $C_{0}$-semigroup.

Theorem 2.1. If $\eta(x)$ satisfies $\bar{\eta}=\sup _{x \in[0, \infty)} \eta(x)<\infty$, then $\mathscr{A}$ generates a positive contraction $C_{0}$ semigroup $T(t)$.

The proof is given in the appendix.

It is easy to check that $X^{*}$, the dual space of $X$, is equal to

$$
X^{*}=\left\{\begin{array}{l|l}
\left(q^{*}, \mathscr{W}^{*}\right) & \begin{array}{l}
q^{*} \in l^{\infty}, \mathscr{W}^{*} \in Y^{*} \\
\left|\left\|\left(q^{*}, \mathscr{W}^{*}\right) \mid\right\|=\sup \left\{\left\|q^{*}\right\|_{l^{\infty}},\left\|\mathscr{W}^{*}\right\|_{Y^{*}}\right\}\right.
\end{array}
\end{array}\right\}
$$

where

$$
Y^{*}=\left\{\begin{array}{l|l}
\mathscr{W}^{*} \mid \begin{array}{l}
\mathscr{W}^{*}=\left(\mathscr{W}_{0}^{*}, \mathscr{W}_{1}^{*}, \cdots\right) \in L^{\infty}[0, \infty) \times L^{\infty}[0, \infty) \times \cdots, \\
\left\|\mathscr{W}^{*}\right\|=\sup _{n \geq 0}\left\|W_{n}^{*}\right\|_{L^{\infty}}<\infty
\end{array}
\end{array}\right\}
$$

It is obvious that $X^{*}$ is a Banach space. If we take a set $\mathrm{Z}$ in $\mathrm{X}$ as

$$
Z=\left\{\begin{array}{l|l}
(p, W) \in X & \begin{array}{l}
p_{n} \geq 0, \quad n \geq 0 \\
W_{n}(x) \geq 0, \quad \forall x \in[0, \infty), \quad n \geq 0,
\end{array}
\end{array}\right\}
$$

then $Z$ is a cone in $X$. For any $(p, W) \in D(\mathscr{A}) \cup Z$, we take

$$
\left(q^{*}, \mathscr{W}^{*}\right)=\|(p, W)\|\left(\left(\begin{array}{c}
1 \\
1 \\
\vdots
\end{array}\right),\left(\begin{array}{c}
1 \\
1 \\
\vdots
\end{array}\right)\right) \in X^{*}
$$

Then, $\left\|\left|q^{*}\|\mid=\| p \|\right.\right.$ and

$$
\left\langle(p, W),\left(q^{*}, \mathscr{W}^{*}\right)\right\rangle=\|(p, W)\| \sum_{n=0}^{\infty} p_{n}+\|(p, W)\| \sum_{n=0}^{\infty} \int_{0}^{\infty} W_{n}(x) d x=\|(p, W)\|^{2},
$$

that is, $\left(q^{*}, \mathscr{W}^{*}\right) \in \theta((p, W)$, where

$$
\theta((p, W))=\left\{( q ^ { * } , \mathscr { W } ^ { * } ) \in X ^ { * } \left|\left\langle(p, W),\left(q^{*}, \mathscr{W}^{*}\right)\right\rangle=\|(p, W)\|^{2}=\left\|\left|\left(q^{*}, \mathscr{W}^{*}\right) \|\right|^{2}\right\}\right.\right.
$$


For such $\left(q^{*}, \mathscr{W}^{*}\right)$, by using boundary condition on $(p, W) \in D(\mathscr{A}) \cup Z$, we calculate

$$
\begin{aligned}
\langle\mathscr{A} & \left.(p, W),\left(q^{*}, \mathscr{W}^{*}\right)\right\rangle \\
= & \|(p, W)\|\left\{-\lambda p_{0}+\int_{0}^{\infty} \eta(x) W_{0}(x) d x\right\} \\
& +\sum_{n=1}^{\infty}\|(p, W)\|\left\{-(\lambda+\xi) p_{n}+\int_{0}^{\infty} \eta(x) W_{n}(x) d x\right\} \\
& +\int_{0}^{\infty}\left\{-\frac{d W_{0}(x)}{d x}-(\lambda+\eta(x)) W_{0}(x)\right\}\|(p, W)\| d x \\
& +\sum_{n=1}^{\infty} \int_{0}^{\infty}\left\{-\frac{d W_{n}(x)}{d x}-(\lambda+\eta(x)) W_{n}(x)+\lambda W_{n-1}(x)\right\}\|(p, W)\| d x \\
= & -\|(p, W)\|\left\{\sum_{n=0}^{\infty} \lambda p_{n}-\sum_{n=1}^{\infty} \xi p_{n}\right\}+\|(p, W)\| \sum_{n=0}^{\infty} \int_{0}^{\infty} \eta(x) W_{n}(x) d x \\
& +\|(p, W)\| \sum_{n=0}^{\infty}\left\{\lambda p_{n}+\xi p_{n+1}\right\}-\|(p, W)\| \sum_{n=0}^{\infty} \int_{0}^{\infty}(\lambda+\eta(x)) W_{n}(x) d x \\
& +\|(p, W)\| \sum_{n=1}^{\infty} \int_{0}^{\infty} \lambda W_{n-1}(x) d x \\
= & 0,
\end{aligned}
$$

which shows that $\mathscr{A}$ is a conservative operator.

From $(p, W)(0) \in D\left(\mathscr{A}^{2}\right) \cup Z$ and the Fattorini theorem (see [12]), we obtain the following result.

Theorem 2.2. $T(t)$ is isometric for the initial value of system (1.7), that is,

$$
\|T(t)(p, W)(0)\|=\|(p, W)(0)\|, \quad \forall t \in[0, \infty) .
$$

From Theorem 2.1 and Theorem 2.2, we obtain the desired results in this paper.

Theorem 2.3. If $\bar{\eta}=\sup _{x \in[0, \infty)} \eta(x)<\infty$, then system (1.7) has a unique positive solution $(p, W)(x, t)$, which satisfies

$$
\|(p, W)(\cdot, t)\|=1, \quad t \in[0, \infty) .
$$

Proof. From Theorem 2.1 and Gupur, Li and Zhu [13], we know that system (1.7) has a unique positive time-dependent solution $(p, W)(x, t)$, which can be expressed as

$$
(p, W)(x, t)=T(t)(p, W)(0), \quad t \in[0, \infty) .
$$

This together with Theorem 2.1 (i.e., (2.1)) gives

$$
\|(p, W)(\cdot, t)\|=\|T(t)(p, W)(0)\|=\|(p, W)(0)\|=1, \quad \forall t \in[0, \infty) .
$$

We remark that (2.2) just reflects the physical background of the model. 


\section{ASYMPTOTIC BEHAVIOR OF SYSTEM (1.7)}

According to Theorem 14 in Gupur, Li and Zhu [13] and using Theorem 2.3, we can obtain the asymptotic behavior of the time-dependent solution of system (1.7). Indeed, we know that the difficulties are the boundary condition. In 1987, Greiner [14] developed an idea through which the spectrum of the underlying operator can be deduced by discussing the maximal operator $A_{m}$ and boundary operators $L$ and $\Phi$.

Now, by using the probability generating function, we prove that 0 is an eigenvalue of $\mathscr{A}$ with geometric multiplicity one.

Lemma 3.1. If $\lambda\left(1+\frac{\lambda}{\xi}\right) \int_{0}^{\infty} x \eta(x) e^{-\int_{0}^{x} \eta_{1}(\tau) d \tau} d x<1$, then 0 is an eigenvalue of $\mathscr{A}$ with geometric multiplicity one.

Proof. We consider the equation $\mathscr{A}\left(p^{(1)}, p^{(2)}\right)=0$, which is equivalent to

$$
\begin{aligned}
\lambda p_{0} & =\int_{0}^{\infty} \eta(x) W_{0}(x) d x, \\
(\lambda+\xi) p_{n} & =\int_{0}^{\infty} \eta(x) W_{n}(x) d x, \quad n \geq 1, \\
\frac{d W_{0}(x)}{d x} & =-(\lambda+\eta(x)) W_{0}(x), \\
\frac{d W_{n}(x)}{d x} & =-(\lambda+\eta(x)) W_{n}(x)+\lambda W_{n-1}(x), \quad n \geq 1, \\
W_{n}(0) & =\lambda p_{n}+\xi p_{n+1}, \quad n \geq 0 .
\end{aligned}
$$

Let the probability generating functions $P(z)=\sum_{n=0}^{\infty} p_{n} z^{n}$, and $G(x, z)=\sum_{n=0}^{\infty} W_{n}(x) z^{n}$, for all complex variables $|z|<1$. Theorem 2.3 ensures that $P(z)$ and $G(x, z)$ are well-defined. (3.1)-(3.2) and (3.3)-(3.4) give

$$
\begin{aligned}
& -\xi p_{0}+\sum_{n=0}^{\infty}(\lambda+\xi) p_{n} z^{n}=\int_{0}^{\infty} \eta(x) \sum_{n=0}^{\infty} W_{n}(x) z^{n} d x \\
& \Longrightarrow \\
& (\lambda+\xi) P(z)=\xi p_{0}+\int_{0}^{\infty} \eta(x) G(x, z) d x, \\
& \sum_{n=0}^{\infty} \frac{\partial W_{n}(x) z^{n}}{d x}=-\sum_{n=0}^{\infty}(\lambda+\eta(x)) W_{n}(x) z^{n}+\lambda z \sum_{n=0}^{\infty} W_{n}(x) z^{n} \\
& \Longrightarrow \\
& \frac{\partial G(x, z)}{\partial x}=(\lambda z-\lambda-\eta(x)) G(x, z) \\
& \Longrightarrow \\
& G(x, z)=G(0, z) e^{\lambda(z-1) x-\int_{0}^{x} \eta(s) d s .}
\end{aligned}
$$


This together with (3.6) and (3.5) follows that

$$
\begin{aligned}
G(0, z) & =\lambda \sum_{n=0}^{\infty} p_{n} z^{n}+\xi \sum_{n=0}^{\infty} p_{n+1} z^{n} \\
& =\lambda P(z)+\frac{\xi}{z} \sum_{n=0}^{\infty} p_{n+1} z^{n+1}=\lambda P(z)+\frac{\xi}{z}\left(\sum_{n=0}^{\infty} p_{n} z^{n}-p_{0}\right) \\
& =\left(\lambda+\frac{\xi}{z}\right)\left[\frac{\xi}{\lambda+\xi} p_{0}+\frac{1}{\lambda+\xi} \int_{0}^{\infty} \eta(x) G(0, z) e^{\lambda(z-1) x-\int_{0}^{x} \eta(s) d s} d x\right]-\frac{\xi}{z} p_{0} \\
& =\frac{\lambda \xi(z-1)}{z(\lambda+\xi)} p_{0}+\frac{\lambda z+\xi}{z(\lambda+\xi)} G(0, z) h(z) \\
& \Longrightarrow \\
G(0, z) & =\frac{\lambda \xi(z-1)}{z(\lambda+\xi)-(\lambda z+\xi) h(z)} p_{0},
\end{aligned}
$$

where $h(z)=\int_{0}^{\infty} \eta(x) e^{\lambda(z-1) x-\int_{0}^{x} \eta(s) d s} d x$. Since $\lim _{z \rightarrow 1} h(z)=\int_{0}^{\infty} \eta(x) e^{-\int_{0}^{x} \eta(s) d s} d x=1$, and $\frac{d h(z)}{d z}=$ $\int_{0}^{\infty} \lambda x \eta(x) e^{\lambda(z-1) x-\int_{0}^{x} \eta(s) d s} d x$, we derive from the L'Hospital rule and (3.8) that

$$
\begin{aligned}
\lim _{z \rightarrow 1} G(0, z) & =\lim _{z \rightarrow 1} \frac{\lambda(z-1) \xi}{z(\lambda+\xi)-(\lambda z+\xi) h(z)} p_{0} \\
& =\lim _{z \rightarrow 1} \frac{\lambda \xi}{\lambda+\xi-\lambda h(z)-(\lambda z+\xi) \frac{d h(z)}{d z}} p_{0} \\
& =\frac{\lambda \xi}{\xi-(\lambda+\xi) \lambda \int_{0}^{\infty} x \eta(x) e^{-\int_{0}^{x} \eta(s) d s} d x} p_{0} \\
& =\frac{\lambda}{1-\lambda\left(1+\frac{\lambda}{\xi}\right) \int_{0}^{\infty} x \eta(x) e^{-\int_{0}^{x} \eta(s) d s} d x} p_{0}
\end{aligned}
$$

By using (3.7), we obtain

$$
\begin{aligned}
\sum_{n=0}^{\infty} W_{n}(x) & =\lim _{z \rightarrow 1} G(x, z)=\frac{\lambda e^{-\int_{0}^{x} \eta(s) d s}}{1-\lambda\left(1+\frac{\lambda}{\xi}\right) \int_{0}^{\infty} x \eta(x) e^{-\int_{0}^{x} \eta(s) d s} d x} p_{0} \\
& \Longrightarrow \\
& \sum_{n=0}^{\infty} \int_{0}^{\infty} W_{n}(x) d x=\frac{\lambda \int_{0}^{\infty} e^{-\int_{0}^{x} \eta(s) d s} d x}{1-\lambda\left(1+\frac{\lambda}{\xi}\right) \int_{0}^{\infty} x \eta(x) e^{-\int_{0}^{x} \eta(s) d s} d x} p_{0}<\infty, \\
\sum_{n=0}^{\infty} p_{n} & =\lim _{z \rightarrow 1} P(z)=\frac{\xi}{\lambda+\xi} p_{0}+\frac{\lambda \int_{0}^{\infty} \eta(x) e^{-\int_{0}^{x} \eta(s) d s} d x}{(\lambda+\xi)\left[1-\lambda\left(1+\frac{\lambda}{\xi}\right) \int_{0}^{\infty} x \eta(x) e^{-\int_{0}^{x} \eta(s) d s} d x\right]} p_{0} \\
& =\frac{\xi}{\lambda+\xi} p_{0}+\frac{\lambda}{(\lambda+\xi)\left[1-\lambda\left(1+\frac{\lambda}{\xi}\right) \int_{0}^{\infty} x \eta(x) e^{-\int_{0}^{x} \eta(s) d s} d x\right]} p_{0}<\infty .
\end{aligned}
$$

(3.9) and (3.10) imply

$$
\|(p, W)\|=\sum_{n=0}^{\infty} p_{n}+\sum_{n=0}^{\infty}\left\|W_{n}\right\|_{L^{1}[0, \infty)}<\infty .
$$

Hence, the eigenvectors corresponding to zero span one-dimensional linear spaces, that is, the geometric multiplicity of 0 is one. 
In the following, by applying Greiner's idea, we obtain the resolvent set of $\mathscr{A}$ on the imaginary axis. To do this, we need to introduce an operator $\left(\mathscr{A}_{0}, D\left(\mathscr{A}_{0}\right)\right)$, and then determine the expression of $\left(\gamma I-\mathscr{A}_{0}\right)^{-1}$. Next, we consider the explicit expression of $\operatorname{ker}\left(\gamma I-A_{m}\right)$ and denote by $D_{\gamma}$ the inverse of $L$ in $\operatorname{ker}\left(\gamma I-A_{m}\right)$, which is called the Dirichlet operator. Moreover, we give the expressions of $D_{\gamma}$ and $\Phi D_{\gamma}$. Finally, by applying the relation between the spectrum of $\mathscr{A}$ and the spectrum of $\Phi D_{\gamma}$, we get the resolvent set of $\mathscr{A}$ on the imaginary axis.

We define $\mathscr{A}_{0}$ and its domain as

$$
\begin{aligned}
\mathscr{A}_{0}(p, W) & =A_{m}(p, W), \\
D\left(\mathscr{A}_{0}\right) & =\left\{(p, W) \in D\left(A_{m}\right) \mid L(p, W)=0\right\},
\end{aligned}
$$

and discuss its inverse. For any given $(y, z) \in X$, we consider the equation $\left(\gamma I-\mathscr{A}_{0}\right)(p, W)=(y, z)$, i.e.,

$$
\begin{aligned}
(\gamma+\lambda) p_{0} & =\int_{0}^{\infty} \eta(x) W_{0}(x) d x+y_{0}, \\
(\gamma+\lambda+\xi) p_{n} & =\int_{0}^{\infty} \eta(x) W_{n}(x) d x+y_{n}, \quad n \geq 1, \\
\frac{d W_{0}(x)}{d x} & =-(\lambda+\eta(x)) W_{0}(x)+z_{0}(x), \\
\frac{d W_{n}(x)}{d x} & =-(\lambda+\eta(x)) W_{n}(x)+\lambda W_{n-1}(x)+z_{n}(x), \quad n \geq 1, \\
W_{n}(0) & =0, \quad n \geq 0 .
\end{aligned}
$$

By solving (3.11)-(3.15), we have

$$
\begin{aligned}
p_{0}= & \frac{1}{\gamma+\lambda} y_{0}+\frac{1}{\gamma+\lambda} \int_{0}^{\infty} \eta(x) W_{0}(x) d x \\
p_{n}= & \frac{1}{\gamma+\lambda+\xi} y_{n}+\frac{1}{\gamma+\lambda+\xi} \int_{0}^{\infty} \eta(x) W_{n}(x) d x \\
W_{0}(x)= & e^{-\int_{0}^{x}(\gamma+\lambda+\eta(s)) d s} \int_{0}^{x} z_{0}(\tau) e^{\int_{0}^{\tau}(\gamma+\lambda+\eta(s)) d s} d \tau, \\
W_{n}(x)= & \lambda e^{-\int_{0}^{x}(\gamma+\lambda+\eta(s)) d s} \int_{0}^{x} W_{n-1}(\tau) e^{\int_{0}^{\tau}(\gamma+\lambda+\eta(s)) d s} d \tau \\
& +e^{-\int_{0}^{x}(\gamma+\lambda+\eta(s)) d s} \int_{0}^{x} z_{n}(\tau) e^{\int_{0}^{\tau}(\gamma+\lambda+\eta(s)) d s} d \tau, \quad n \geq 1 .
\end{aligned}
$$

If we introduce the following operators

$$
V f(x)=e^{-\int_{0}^{x}(\gamma+\lambda+\eta(s)) d s} \int_{0}^{x} f(\tau) e^{\int_{0}^{\tau}(\gamma+\lambda+\eta(s)) d s} d \tau, \quad f \in L^{1}[0, \infty)
$$

then (3.18) and (3.19) imply

$$
\begin{aligned}
W_{0}(x) & =V z_{0}(x), \\
W_{n}(x) & =\sum_{k=0}^{n} \lambda^{k} V^{k+1} z_{n-k}(x), \quad n \geq 1, \\
p_{0} & =\frac{1}{\gamma+\lambda} y_{0}+\frac{1}{\gamma+\lambda} \psi V z_{0}(x), \\
p_{n} & =\frac{1}{\gamma+\lambda+\xi} y_{n}+\frac{1}{\gamma+\lambda+\xi} \sum_{k=0}^{n} \lambda^{k} \psi V^{k+1} z_{n-k}(x) .
\end{aligned}
$$


(3.20)-(3.23) give the expression of $\left(\gamma I-\mathscr{A}_{0}\right)^{-1}$ as follows if $\left(\gamma I-\mathscr{A}_{0}\right)^{-1}$ exists

$$
\begin{aligned}
\left(\gamma I-\mathscr{A}_{0}\right)^{-1} & \left.\left(\begin{array}{c}
y_{0} \\
y_{1} \\
y_{2} \\
y_{3} \\
\vdots
\end{array}\right),\left(\begin{array}{c}
z_{0}(x) \\
z_{1}(x) \\
z_{2}(x) \\
z_{3}(x) \\
\vdots
\end{array}\right)\right)=\left(\begin{array}{ccccc}
\frac{1}{\gamma+\lambda} & 0 & 0 & 0 & \ldots \\
0 & \frac{1}{\gamma+\lambda+\xi} & 0 & 0 & \ldots \\
0 & 0 & \frac{1}{\gamma+\lambda+\xi} & 0 & \ldots \\
0 & 0 & 0 & \frac{1}{\gamma+\lambda+\xi} & \ldots \\
\vdots & \vdots & \vdots & \vdots & \ddots
\end{array}\right)\left(\begin{array}{c}
y_{0} \\
y_{1} \\
y_{2} \\
y_{3} \\
\vdots
\end{array}\right) \\
+ & \left(\begin{array}{ccccc}
\frac{1}{\gamma+\lambda} \psi V & 0 & 0 & 0 & \ldots \\
\frac{\lambda}{\gamma+\lambda+\xi} \psi V^{2} & \frac{1}{\gamma+\lambda+\xi} \psi V & 0 & 0 & \ldots \\
\frac{\lambda^{2}}{\gamma+\lambda+\xi} \psi V^{3} & \frac{\lambda}{\gamma+\lambda+\xi} \psi V^{2} & \frac{1}{\gamma+\lambda+\xi} \psi V & 0 & \ldots \\
\frac{\lambda^{3}}{\gamma+\lambda+\xi} \psi V^{4} & \frac{\lambda^{2}}{\gamma+\lambda+\xi} \psi V^{3} & \frac{\lambda}{\gamma+\lambda+\xi} \psi V^{2} & \frac{1}{\gamma+\lambda+\xi} \psi V & \ldots \\
\vdots & \vdots & \vdots & \ddots
\end{array}\right)\left(\begin{array}{c}
z_{0}(x) \\
z_{1}(x) \\
z_{2}(x) \\
z_{3}(x) \\
\vdots
\end{array}\right), \\
& \left.\left(\begin{array}{ccccc}
V & 0 & 0 & 0 & \ldots \\
\lambda V^{2} & V & 0 & 0 & \ldots \\
\lambda^{2} V^{3} & \lambda V^{2} & V & 0 & \ldots \\
\lambda^{3} V^{4} & \lambda^{2} V^{3} & \lambda V^{2} & V & \ldots \\
\vdots & \vdots & \vdots & \vdots & \ddots
\end{array}\right)\left(\begin{array}{c}
z_{0}(x) \\
z_{1}(x) \\
z_{2}(x) \\
z_{3}(x) \\
\vdots
\end{array}\right)\right)
\end{aligned}
$$

From which together with the definition of the resolvent set we have the following result.

Lemma 3.2. If

$$
0<\underline{\eta}=\inf _{x \in[0, \infty)} \eta(x) \leq \bar{\eta}=\sup _{x \in[0, \infty)} \eta(x)<\infty
$$

then

$$
\{\gamma \in \mathbb{C} \mid \operatorname{Re} \gamma+\lambda>0, \operatorname{Re} \gamma+\underline{\eta}>0\} \subset \rho\left(\mathscr{A}_{0}\right) .
$$

That is, all points on the imaginary axis belong to the resolvent set of $\mathscr{A}_{0}$.

Proof. For any $f \in L^{1}[0, \infty)$, we estimate

$$
\begin{aligned}
\|V f\|_{L^{1}[0, \infty)} \leq & \int_{0}^{\infty} \frac{-1}{\operatorname{Re} \gamma+\lambda+\underline{\eta}} \int_{0}^{x}|f(\tau)| e^{\int_{0}^{\tau}(\operatorname{Re} \gamma+\lambda+\eta(s)) d s} d e^{-\int_{0}^{x}(\operatorname{Re} \gamma+\lambda+\eta(s)) d s} \\
= & -\frac{1}{\operatorname{Re} \gamma+\lambda+\underline{\eta}}\left\{\left.e^{-\int_{0}^{x}(\operatorname{Re} \gamma+\lambda+\eta(s)) d s} \int_{0}^{x}|f(\tau)| e^{\int_{0}^{\tau}(\operatorname{Re} \gamma+\lambda+\eta(s)) d s} d \tau\right|_{x=0} ^{x=\infty}\right. \\
& \left.-\int_{0}^{\infty}|f(x)| e^{\int_{0}^{x}(\operatorname{Re} \gamma+\lambda+\eta(s)) d s} e^{-\int_{0}^{x}(\operatorname{Re} \gamma+\lambda+\eta(s)) d s} d x\right\} \\
= & \frac{1}{\operatorname{Re} \gamma+\lambda+\underline{\eta}}\|f\|_{L^{1}[0, \infty)} \\
& \Longrightarrow \\
\|V\| \leq & \frac{1}{\operatorname{Re} \gamma+\lambda+\underline{\eta}} .
\end{aligned}
$$


By using $\|\phi\| \leq \bar{\eta}$ and (3.24) we estimate, for any $(y, z) \in X$

$$
\begin{aligned}
& \left\|\left(\gamma I-\mathscr{A}_{0}\right)^{-1}(y, z)\right\| \\
\leq & \left|\frac{1}{\gamma+\lambda} y_{0}+\frac{1}{\gamma+\lambda} \psi V z_{0}\right|+\left|\frac{1}{\gamma+\lambda+\xi} y_{1}+\frac{\lambda}{\gamma+\lambda+\xi} \psi V^{2} z_{0}+\frac{1}{\gamma+\lambda+\xi} \psi V z_{1}\right| \\
& +\mid \frac{1}{\gamma+\lambda+\xi} y_{2}+\frac{\lambda^{2}}{\gamma+\lambda+\xi} \psi V^{3} z_{0}+\frac{\lambda}{\gamma+\lambda+\xi} \psi V^{2} z_{1} \\
& +\frac{1}{\gamma+\lambda+\xi} \psi V z_{2} \mid+\cdots+\left\|V z_{0}\right\|_{L^{1}[0, \infty)}+\left\|\lambda V^{2} z_{0}+V z_{1}\right\|_{L 1[0, \infty)} \\
& +\left\|\lambda^{2} V^{3} z_{0}+\lambda V^{2} z_{1}+V z_{2}\right\|_{L^{1}[0, \infty)}+\left\|\sum_{k=0}^{3} \lambda^{k} V^{k+1} z_{3-k}\right\|_{L^{1}[0, \infty)} \\
& +\cdots+\|\left.\sum_{k=0}^{n} \lambda^{k} V^{k+1} z_{n-k}||\right|_{L^{1}[0, \infty)}+\cdots \\
\leq & \frac{1}{|\gamma+\lambda|}\left|y_{0}\right|+\frac{1}{|\gamma+\lambda+\xi|} \sum_{n=1}^{\infty}\left|y_{n}\right| \\
& +\frac{1}{|\gamma+\lambda+\xi|} \sum_{n=0}^{\infty} \sum_{k=0}^{n} \lambda^{k}\|\psi\|\|V\|^{k+1}\left\|z_{n-k}\right\|_{L^{1}[0, \infty)}+\sum_{n=0}^{\infty} \sum_{k=0}^{n} \lambda^{k}\|V\|^{k+1}\left\|z_{n-k}\right\|_{L^{1}[0, \infty)} \\
\leq & \sup \left\{\frac{1}{|\gamma+\lambda|}, \frac{1}{|\gamma+\lambda+\xi|(\operatorname{Re} \gamma+\underline{\eta})}+\frac{1}{\operatorname{Re} \gamma+\underline{\eta}}\right\}\|(y, z)\| \\
<\infty . &
\end{aligned}
$$

This completes the proof.

\section{Lemma 3.3. Let}

$$
0<\underline{\eta}=\inf _{x \in[0, \infty)} \eta(x) \leq \bar{\eta}=\sup _{x \in[0, \infty)} \eta(x)<\infty .
$$

If $\gamma \in \rho\left(\mathscr{A}_{0}\right)$, then

$$
\begin{aligned}
& (p, W) \in \operatorname{ker}\left(\gamma I-A_{m}\right) \Longleftrightarrow \\
& p_{0}=\frac{1}{\gamma+\lambda} a_{0} \int_{0}^{\infty} \eta(x) e^{-\int_{0}^{x}(\gamma+\lambda+\eta(s)) d s} d x \\
& p_{n}=\frac{1}{\gamma+\lambda+\xi} \int_{0}^{\infty} \eta(x) e^{-\int_{0}^{x}(\gamma+\lambda+\eta(s)) d s} \sum_{k=0}^{n} \frac{(\lambda x)^{k}}{k !} a_{n-k} d x, \quad n \geq 1, \\
& W_{0}(x)=a_{0} e^{-\int_{0}^{x}(\gamma+\lambda+\eta(s)) d s} \\
& W_{n}(x)=e^{-\int_{0}^{x}(\lambda+\eta(s)) d s} \sum_{k=0}^{n} \frac{(\lambda x)^{k}}{k !} a_{n-k}, \quad n \geq 1, \\
& \left(a_{0}, a_{1}, a_{2}, \cdots\right) \in l_{1}
\end{aligned}
$$


Proof. If $(p, W) \in \operatorname{ker}\left(\gamma I-A_{m}\right)$, then $\left(\gamma I-A_{m}\right)(p, W)=0$, which is equivalent to

$$
\begin{aligned}
(\gamma+\lambda) p_{0} & =\int_{0}^{\infty} \eta(x) W_{0}(x) d x, \\
(\gamma+\lambda+\xi) p_{n} & =\int_{0}^{\infty} \eta(x) W_{n}(x) d x, \quad n \geq 1, \\
\frac{d W_{0}(x)}{d x} & =-(\gamma+\lambda+\eta(x)) W_{0}(x), \\
\frac{d W_{n}(x)}{d x} & =-(\gamma+\lambda+\eta(x)) W_{n}(x)+\lambda W_{n-1}(x), \quad n \geq 1 .
\end{aligned}
$$

By solving (3.28)-(3.29), we have

$$
\begin{aligned}
& W_{0}(x)=a_{0} e^{-\int_{0}^{x}(\gamma+\lambda+\eta(s)) d s}, \\
& W_{n}(x)=a_{n} e^{-\int_{0}^{x}(\gamma+\lambda+\eta(s)) d s}+\lambda e^{-\int_{0}^{x}(\gamma+\lambda+\eta(s)) d s} \int_{0}^{x} W_{n-1}(\tau) e^{\int_{0}^{\tau}(\gamma+\lambda+\eta(s)) d s} d \tau, n \geq 1 .
\end{aligned}
$$

Repeating application of (3.30) and (3.31) yields

$$
\begin{aligned}
W_{1}(x) & =a_{1} e^{-\int_{0}^{x}(\gamma+\lambda+\eta(s)) d s}+\lambda e^{-\int_{0}^{x}(\gamma+\lambda+\eta(s)) d s} \int_{0}^{x} a_{0} d \tau \\
& =e^{-\int_{0}^{x}(\gamma+\lambda+\eta(s)) d s}\left[a_{1}+\lambda x a_{0}\right], \\
W_{2}(x) & =a_{2} e^{-\int_{0}^{x}(\gamma+\lambda+\eta(s)) d s}+\lambda e^{-\int_{0}^{x}(\gamma+\lambda+\eta(s)) d s} \int_{0}^{x}\left[a_{1}+\lambda \tau a_{0}\right] d \tau \\
& =e^{-\int_{0}^{x}(\gamma+\lambda+\eta(s)) d s}\left[a_{2}+\lambda x a_{1}+\frac{(\lambda x)^{2}}{2} a_{0}\right], \\
& \ldots \ldots \\
W_{n}(x) & =e^{-\int_{0}^{x}(\gamma+\lambda+\eta(s)) d s}\left[a_{n}+\lambda x a_{n-1}+\frac{(\lambda x)^{2}}{2} a_{n-2}+\cdots+\frac{(\lambda x)^{n}}{n !} a_{0}\right] \\
& =e^{-\int_{0}^{x}(\gamma+\lambda+\eta(s)) d s} \sum_{k=0}^{n} \frac{(\lambda x)^{k}}{k !} a_{n-k}, \quad n \geq 1 .
\end{aligned}
$$

Substituting (3.30) and (3.34) into (3.26) and (3.27), we derive

$$
\begin{aligned}
& p_{0}=\frac{1}{\gamma+\lambda} a_{0} \int_{0}^{\infty} \eta(x) e^{-\int_{0}^{x}(\gamma+\lambda+\eta(s)) d s} d x \\
& p_{n}=\frac{1}{\gamma+\lambda+\xi} \int_{0}^{\infty} \eta(x) e^{-\int_{0}^{x}(\gamma+\lambda+\eta(s)) d s} \sum_{k=0}^{n} \frac{(\lambda x)^{k}}{k !} a_{n-k} d x, \quad n \geq 1
\end{aligned}
$$

Since $(p, W) \in \operatorname{ker}\left(\gamma I-D\left(A_{m}\right)\right),(p, W) \in D\left(A_{m}\right)$ implies by the imbedding theorem in Adams [15] that

$$
\begin{aligned}
\sum_{n=0}^{\infty}\left|a_{n}\right| & =\sum_{n=0}^{\infty}\left|W_{n}(0)\right| \leq \sum_{n=0}^{\infty}\left\|W_{n}\right\|_{L^{\infty}[0, \infty)} \\
& \leq \sum_{n=0}^{\infty}\left\|W_{n}\right\|_{L^{1}[0, \infty)}+\sum_{n=0}^{\infty}\left\|\frac{d W_{n}}{d x}\right\|_{L^{1}[0, \infty)}<\infty
\end{aligned}
$$


Hence, (3.30), (3.34), (3.35), (3.36) and (3.37) are necessary for this lemma. Conversely, if (3.25) hold, then by direct calculating, we have

$$
\begin{aligned}
\frac{d W_{0}(x)}{d x}= & -(\gamma+\lambda+\eta(x)) a_{0} e^{-\int_{0}^{x}(\gamma+\lambda+\eta(s)) d s} \\
= & -(\gamma+\lambda+\eta(x)) W_{0}(x) \\
\frac{d W_{n}(x)}{d x}= & -(\gamma+\lambda+\eta(x)) e^{-\int_{0}^{x}(\gamma+\lambda+\eta(s)) d s} \sum_{k=0}^{n} \frac{(\lambda x)^{k}}{k !} a_{n-k} \\
& +e^{-\int_{0}^{x}(\gamma+\lambda+\eta(s)) d s} \sum_{k=1}^{n} \frac{\lambda^{k} x^{k-1}}{(k-1) !} a_{n-k} \\
= & -(\gamma+\lambda+\eta(x)) W_{n}(x)+\lambda e^{-\int_{0}^{x}(\gamma+\lambda+\eta(s)) d s} \sum_{l=0}^{n-1} \frac{(\lambda x)^{l}}{l !} a_{n-1-l} \\
= & -(\gamma+\lambda+\eta(x)) W_{n}(x)+\lambda W_{n-1}(x), \quad n \geq 1 .
\end{aligned}
$$

By using $\int_{0}^{\infty} x^{k} e^{-b x} d x=\frac{k !}{b^{k+1}}, \quad k \geq 1, b>0$, we estimate

$$
\begin{aligned}
\sum_{n=0}^{\infty}\left\|W_{n}\right\|_{L^{1}[0, \infty)} & \leq \sum_{n=0}^{\infty} \sum_{k=0}^{n}\left|a_{n-k}\right| \frac{\lambda^{k}}{k !} \int_{0}^{\infty} x^{k} e^{-\int_{0}^{x}(\operatorname{Re} \gamma+\lambda+\underline{\eta}) d s} d x \\
& =\sum_{n=0}^{\infty} \sum_{k=0}^{n}\left|a_{n-k}\right| \frac{\lambda^{k}}{k !} \frac{k !}{(\operatorname{Re} \gamma+\lambda+\underline{\eta})^{k+1}} \\
& =\sum_{k=0}^{\infty} \frac{\lambda^{k}}{(\operatorname{Re} \gamma+\lambda+\underline{\eta})^{k+1}} \sum_{n=k}^{\infty}\left|a_{n-k}\right| \\
& \leq \frac{1}{\operatorname{Re} \gamma+\underline{\eta}} \sum_{n=0}^{\infty}\left|a_{n}\right|<\infty .
\end{aligned}
$$

(3.38), (3.39) and (3.40) give

$$
\begin{aligned}
\sum_{n=0}^{\infty}\left\|\frac{d W_{n}}{d x}\right\|_{L^{1}[0, \infty)} & \leq(\operatorname{Re} \gamma+\lambda+\bar{\eta}) \sum_{n=0}^{\infty}\left\|W_{n}\right\|_{L^{1}[0, \infty)}+\lambda \sum_{n=1}^{\infty}\left\|W_{n-1}\right\|_{L^{1}[0, \infty)} \\
& \leq\left(\frac{\operatorname{Re} \gamma+\lambda+\bar{\eta}}{\operatorname{Re} \gamma+\underline{\eta}}+\frac{\lambda}{\operatorname{Re} \gamma+\underline{\eta}}\right) \sum_{n=0}^{\infty}\left|a_{n}\right|<\infty
\end{aligned}
$$

(3.38)-(3.41) show that $(p, W) \in \operatorname{ker}\left(\gamma I-A_{m}\right)$.

Observe that the operator $L$ is surjective. So,

$$
\left.L\right|_{\operatorname{ker}\left(\gamma I-A_{m}\right)}: \operatorname{ker}\left(\gamma I-A_{m}\right) \rightarrow \partial X
$$

is invertible if $\gamma \in \rho\left(\mathscr{A}_{0}\right)$. Thus, we introduce an operator $D_{\gamma}$ as follows

$$
D_{\gamma}=\left(\left.L\right|_{\operatorname{ker}\left(\gamma I-A_{m}\right)}\right)^{-1}: \partial X \rightarrow \operatorname{ker}\left(\gamma I-A_{m}\right)
$$


Lemma 3.3 gives the explicit form of $D_{\gamma}$ for all $\gamma \in \rho\left(\mathscr{A}_{0}\right)$,

$$
\begin{aligned}
\left(\begin{array}{c}
a_{0} \\
a_{1} \\
a_{2} \\
a_{3} \\
\vdots
\end{array}\right)= & \left(\left(\begin{array}{ccccc}
\frac{1}{\gamma+\lambda} \psi m_{11} & 0 & 0 & \ldots \\
\frac{1}{\gamma+\lambda+\xi} \psi m_{21} & \frac{1}{\gamma+\lambda+\xi} \psi m_{22} & 0 & \ldots \\
\frac{1}{\gamma+\lambda+\xi} \psi m_{31} & \frac{1}{\gamma+\lambda+\xi} \psi m_{32} & \frac{1}{\gamma+\lambda+\xi} \psi m_{33} & \ldots \\
\vdots & & \vdots & \vdots & \ddots
\end{array}\right)\left(\begin{array}{c}
a_{0} \\
a_{1} \\
a_{2} \\
a_{3} \\
\vdots
\end{array}\right),\right. \\
& \left.\left(\begin{array}{ccccc}
m_{11} & 0 & 0 & 0 & \ldots \\
m_{21} & m_{22} & 0 & 0 & \ldots \\
m_{31} & m_{32} & m_{33} & 0 & \ldots \\
m_{41} & m_{42} & m_{43} & m_{44} & \ldots \\
\vdots & \vdots & \vdots & \vdots & \ddots
\end{array}\right)\left(\begin{array}{c}
a_{0} \\
a_{1} \\
a_{2} \\
a_{3} \\
\vdots
\end{array}\right)\right)
\end{aligned}
$$

where

$$
m_{i j}=\frac{(\lambda x)^{i-j}}{(i-j) !} e^{-\int_{0}^{x}(\gamma+\lambda+\eta(s)) d s}, \quad i=1,2, \cdots, j=1,2, \cdots, i
$$

From the expression of $D_{\gamma}$ and the definition of $\Phi$, it is not difficult to determine the explicit form of $\Phi D_{\gamma}$ as follows

$$
\begin{aligned}
\Phi D_{\gamma}\left(\begin{array}{c}
a_{0} \\
a_{1} \\
a_{2} \\
a_{3} \\
\vdots
\end{array}\right)= & \left(\begin{array}{cccc}
\frac{\lambda}{\gamma+\lambda} \psi m_{11} & 0 & 0 & \ldots \\
\frac{\lambda}{\gamma+\lambda+\xi} \psi m_{21} & \frac{\lambda}{\gamma+\lambda+\xi} \psi m_{22} & 0 & \ldots \\
\frac{\lambda}{\gamma+\lambda+\xi} \psi m_{31} & \frac{\lambda}{\gamma+\lambda+\xi} \psi m_{32} & \frac{\lambda}{\gamma+\lambda+\xi} \psi m_{33} & \ldots \\
\vdots & \vdots & \vdots & \ddots
\end{array}\right)\left(\begin{array}{c}
a_{0} \\
a_{1} \\
a_{2} \\
a_{3} \\
\vdots
\end{array}\right) \\
& \left.+\left(\begin{array}{ccccc}
\frac{\xi}{\gamma+\lambda+\xi} \psi m_{21} & \frac{\xi}{\gamma+\lambda+\xi} \psi m_{22} & 0 & 0 & \ldots \\
\frac{\xi}{\gamma+\lambda+\xi} \psi m_{31} & \frac{\xi}{\gamma+\lambda+\xi} \psi m_{32} & \frac{\xi}{\gamma+\lambda+\xi} \psi m_{33} & 0 & \ldots \\
\frac{\xi}{\gamma+\lambda+\xi} \psi m_{41} & \frac{\xi}{\gamma+\lambda+\xi} \psi m_{42} & \frac{\xi}{\gamma+\lambda+\xi} \psi m_{43} & \frac{\xi}{\gamma+\lambda+\xi} \psi m_{44} & \ldots \\
\vdots & \vdots & \vdots & \vdots & \ddots
\end{array}\right)\left(\begin{array}{c}
a_{0} \\
a_{1} \\
a_{2} \\
a_{3} \\
\vdots
\end{array}\right)\right) .
\end{aligned}
$$

According to Haji and Radl [16], we know that the following lemma illustrates the relations between the spectrum of $\mathscr{A}$ and spectrum of $\Phi D_{\gamma}$.

Lemma 3.4. If $\gamma \in \rho\left(\mathscr{A}_{0}\right)$ and there exists $\gamma_{0} \in \mathbb{C}$ such that $1 \notin \sigma\left(\Phi D_{\gamma_{0}}\right)$, then

$$
\gamma \in \sigma(\mathscr{A}) \Longleftrightarrow 1 \in \sigma\left(\Phi D_{\gamma}\right) \text {. }
$$

From Lemma 3.4 and Nagel [17], we obtain the resolvent set of $\mathscr{A}$ on the imaginary axis.

Lemma 3.5. If $0<\underline{\eta}=\inf _{x \in[0, \infty)} \eta(x) \leq \bar{\eta}=\sup _{x \in[0, \infty)} \eta(x)<\infty$, then all points on the imaginary axis except zero belong to the resolvent set of $\mathscr{A}$.

Proof. Let $\gamma=i \omega, \omega \in \mathbb{R} \backslash\{0\}$. The Riemann-Lebesgue lemma

$$
\lim _{\omega \rightarrow \infty} \int_{0}^{\infty} f(x) \cos \omega x d x=0, \lim _{\omega \rightarrow \infty} \int_{0}^{\infty} f(x) \sin \omega x d x=0, f \in L^{1}[0, \infty)
$$


implies that there exists a positive constant $\Theta>0$ such that, $\forall|\omega|>\Theta$,

$$
\begin{aligned}
\left|\int_{0}^{\infty} f(x) e^{-i \omega x} d x\right|^{2} & =\left|\int_{0}^{\infty} f(x)(\cos \omega x-i \sin \omega x) d x\right|^{2} \\
& <\left(\int_{0}^{\infty} f(x) d x\right)^{2}, \quad 0<f \in L^{1}[0, \infty)
\end{aligned}
$$

In this formula, by replacing $f(x)$ with $\eta(x) e^{-\int_{0}^{x}(\lambda+\eta(s)) d s}$, and using the fact $\int_{0}^{\infty} \eta(x) e^{-\int_{0}^{x} \eta(s) d s} d x=1$, and

$$
\begin{aligned}
& \sum_{j=l}^{\infty}\left|\int_{0}^{\infty} \eta(x) \frac{(\lambda x)^{j-l}}{(j-l) !} e^{-\int_{0}^{x}(i \omega+\lambda+\eta(s)) d s} d x\right| \\
& \leq \sum_{j=l}^{\infty} \int_{0}^{\infty} \eta(x) \frac{(\lambda x)^{j-1}}{(j-l) !}\left|e^{-i \omega x}\right|\left|e^{-\int_{0}^{x}(\lambda+\eta(s)) d s}\right| d x \\
& =\sum_{j=l}^{\infty} \int_{0}^{\infty} \eta(x) \frac{(\lambda x)^{j-1}}{(j-l) !} e^{-\int_{0}^{x}(\lambda+\eta(s)) d s} d x, \quad l \geq 1 .
\end{aligned}
$$

we estimate, for $\vec{a}=\left(a_{0}, a_{1}, a_{2}, \cdots\right) \in l^{1}$,

$$
\begin{aligned}
& \left\|\Phi D_{\gamma}(\vec{a})\right\| \leq\left|\frac{\lambda}{\gamma+\lambda} \psi m_{11}+\frac{\lambda}{\gamma+\lambda+\xi} \sum_{j=2}^{\infty} \psi m_{j 1}+\frac{\xi}{\gamma+\lambda+\xi} \sum_{j=2}^{\infty} \psi m_{j 1}\right|\left|a_{0}\right| \\
& +\left|\frac{\lambda}{\gamma+\lambda+\xi} \sum_{j=2}^{\infty} \psi m_{j 2}+\frac{\xi}{\gamma+\lambda+\xi} \sum_{j=2}^{\infty} \psi m_{j 2}\right|\left|a_{1}\right| \\
& +\left|\frac{\lambda}{\gamma+\lambda+\xi} \sum_{j=3}^{\infty} \psi m_{j 3}+\frac{\xi}{\gamma+\lambda+\xi} \sum_{j=3}^{\infty} \psi m_{j 3}\right|\left|a_{2}\right| \\
& +\left|\frac{\lambda}{\gamma+\lambda+\xi} \sum_{j=4}^{\infty} \psi m_{j 4}+\frac{\xi}{\gamma+\lambda+\xi} \sum_{j=4}^{\infty} \psi m_{j 4}\right|\left|a_{3}\right|+\cdots \\
& \leq \frac{\lambda}{|\gamma+\lambda|}\left|\psi m_{11}\right|\left|a_{0}\right|+\frac{\lambda+\xi}{|\gamma+\lambda+\xi|} \sum_{j=2}^{\infty}\left|\psi m_{j 1}\right|\left|a_{0}\right| \\
& +\frac{\lambda+\xi}{|\gamma+\lambda+\xi|} \sum_{j=2}^{\infty}\left|\psi m_{j 2}\right| a_{1}\left|+\frac{\lambda+\xi}{|\gamma+\lambda+\xi|} \sum_{j=3}^{\infty}\right| \psi m_{j 3}|| a_{2} \mid \\
& +\frac{\lambda+\xi}{|\gamma+\lambda+\xi|} \sum_{j=4}^{\infty}\left|\psi m_{j 4}\right|\left|a_{3}\right|+\cdots \\
& <\int_{0}^{\infty} \eta(x) \sum_{j=1}^{\infty} \frac{(\lambda x)^{j-1}}{(j-1) !} e^{-\int_{0}^{x}(\lambda+\eta(s)) d s} d x\left|a_{0}\right|+\int_{0}^{\infty} \eta(x) \sum_{j=2}^{\infty} \frac{(\lambda x)^{j-2}}{(j-2) !} e^{-\int_{0}^{x}(\lambda+\eta(s)) d s} d x\left|a_{1}\right| \\
& +\int_{0}^{\infty} \eta(x) \sum_{j=3}^{\infty} \frac{(\lambda x)^{j-3}}{(j-3) !} e^{-\int_{0}^{x}(\lambda+\eta(s)) d s} d x\left|a_{2}\right|+\int_{0}^{\infty} \eta(x) \sum_{j=4}^{\infty} \frac{(\lambda x)^{j-4}}{(j-4) !} e^{-\int_{0}^{x}(\lambda+\eta(s)) d s} d x\left|a_{3}\right|+\cdots \\
& =\int_{0}^{\infty} \eta(x) e^{-\int_{0}^{x} \eta(s) d s} d x \sum_{n=0}^{\infty}\left|a_{n}\right|=\sum_{n=0}^{\infty}\left|a_{n}\right|=\|\vec{a}\| .
\end{aligned}
$$

Thus, $\left\|\Phi D_{\gamma}\right\|<1$, which means that $1 \notin \sigma\left(\Phi D_{\gamma}\right)$ when $|\omega|>\Theta$. This together with Lemma 3.4 gives $\gamma \in \rho(\mathscr{A})$ for $|\omega|>\Theta$, i.e.,

$$
\sigma(\mathscr{A}) \cap i \mathbb{R} \subset\{i \omega|| \omega \mid \leq \Theta\}
$$


is bounded. From Theorem 2.3 and Lemma 3.1, we know that spectral bound $s(\mathscr{A})=0$. Using Nagel [17, P302], we obtain that $\sigma(\mathscr{A})$ is imaginary additively cyclic, which states that

$$
i \omega \in \sigma(\mathscr{A}) \Rightarrow i \omega k \in \sigma(\mathscr{A}), \quad \text { all positive integer } k \text {. }
$$

Now, the boundedness of $\sigma(\mathscr{A}) \cap i \mathbb{R}$ gives $i \mathbb{R} \cap \sigma(\mathscr{A})=\{0\}$.

In the following, we give the explicit expression of $\mathscr{A}^{*}$, the adjoint operator of $\mathscr{A}$, and obtain that 0 is an eigenvalue of $\mathscr{A}^{*}$ with geometric multiplicity one.

\section{Lemma 3.6.}

$$
\begin{aligned}
& \mathscr{A}^{*}\left(q^{*}, \mathscr{W}^{*}\right)=\left(\left(\begin{array}{ccccc}
-\lambda & 0 & 0 & 0 & \ldots \\
0 & -(\lambda+\xi) & & 0 & \ldots \\
0 & 0 & -(\lambda+\xi) & 0 & \ldots \\
0 & 0 & 0 & -(\lambda+\xi) & \ldots \\
\vdots & \vdots & \vdots & \vdots & \ddots
\end{array}\right)\left(\begin{array}{c}
q_{0}^{*} \\
q_{1}^{*} \\
q_{2}^{*} \\
q_{3}^{*} \\
\vdots
\end{array}\right)\right. \\
& +\left(\begin{array}{ccccc}
\lambda & 0 & 0 & 0 & \cdots \\
\xi & \lambda & 0 & 0 & \cdots \\
0 & \xi & \lambda & 0 & \cdots \\
0 & 0 & \xi & \lambda & \cdots \\
\vdots & \vdots & \vdots & \vdots & \ddots
\end{array}\right)\left(\begin{array}{c}
\mathscr{W}_{0}^{*}(0) \\
\mathscr{W}_{1}^{*}(0) \\
\mathscr{W}_{2}^{*}(0) \\
\mathscr{W}_{3}^{*}(0) \\
\vdots
\end{array}\right) \\
& \left(\begin{array}{ccccc}
\frac{d}{d x}-(\lambda+\eta(x)) & \lambda & 0 & 0 & \ldots \\
0 & \frac{d}{d x}-(\lambda+\eta(x)) & \lambda & 0 & \ldots \\
0 & 0 & \frac{d}{d x}-(\lambda+\eta(x)) & \lambda & \ldots \\
\vdots & \vdots & \vdots & \vdots & \ddots
\end{array}\right)\left(\begin{array}{c}
\mathscr{W}_{0}^{*}(x) \\
\mathscr{W}_{1}^{*}(x) \\
\mathscr{W}_{2}^{*}(x) \\
\vdots
\end{array}\right) \\
& \left.+\left(\begin{array}{cccc}
\eta(x) & 0 & 0 & \cdots \\
0 & \eta(x) & 0 & \cdots \\
0 & 0 & \eta(x) & \cdots \\
\vdots & \vdots & \vdots & \ddots
\end{array}\right)\left(\begin{array}{c}
q_{0}^{*} \\
q_{1}^{*} \\
q_{2}^{*} \\
\vdots
\end{array}\right)\right) \\
& D\left(\mathscr{A}^{*}\right)=\left\{\left(q^{*}, \mathscr{W}^{*}\right) \in X^{*} \mid \frac{d \mathscr{W}_{n}^{*}(x)}{d x} \text { exist and } \mathscr{W}_{n}^{*}(\infty)=\alpha, n \geq 0\right\}
\end{aligned}
$$

where $\alpha$ in $D\left(\mathscr{A}^{*}\right)$ is a constant which is irrelevant to $n$. 
Proof. By using integration by parts and the boundary conditions on $(p, W) \in D(\mathscr{A})$, we have, for $\left(q^{*}, \mathscr{W}^{*}\right) \in D\left(\mathscr{A}^{*}\right)$

$$
\begin{aligned}
\left\langle\mathscr{A}(p, W),\left(q^{*}, \mathscr{W}^{*}\right)\right\rangle= & \left\{-\lambda p_{0}+\int_{0}^{\infty} \eta(x) W_{0}(x) d x\right\} q_{0}^{*}+\sum_{n=1}^{\infty}\left\{-(\lambda+\xi) p_{n}+\int_{0}^{\infty} \eta(x) W_{n}(x) d x\right\} q_{n}^{*} \\
& +\int_{0}^{\infty}\left\{-\frac{d W_{0}(x)}{d x}-(\lambda+\eta(x)) W_{0}(x)\right\} \mathscr{W}_{0}^{*}(x) d x \\
& +\sum_{n=1}^{\infty} \int_{0}^{\infty}\left\{-\frac{d W_{n}(x)}{d x}-(\lambda+\eta(x)) W_{n}(x)+\lambda W_{n-1}(x)\right\} \mathscr{W}_{n}^{*}(x) d x \\
= & p_{0}\left(-\lambda q_{0}^{*}\right)+\sum_{n=1}^{\infty} p_{n}\left[-(\lambda+\xi) q_{n}^{*}\right]+\sum_{n=0}^{\infty} \int_{0}^{\infty} W_{n}(x)\left[\eta(x) q_{n}^{*}\right] d x \\
& +\sum_{n=0}^{\infty} W_{n}(0) \mathscr{W}_{n}^{*}(0)+\sum_{n=0}^{\infty} \int_{0}^{\infty} W_{n}(x) \frac{d \mathscr{W}_{n}^{*}(x)}{d x} d x \\
& +\sum_{n=0}^{\infty} \int_{0}^{\infty} W_{n}(x)\left[-(\lambda+\eta(x)) \mathscr{W}_{n}^{*}(x)\right] d x+\sum_{n=0}^{\infty} \int_{0}^{\infty} W_{n}(x)\left[\lambda \mathscr{W}_{n+1}^{*}(x)\right] d x \\
= & p_{0}\left(-\lambda q_{0}^{*}\right)+\sum_{n=1}^{\infty} p_{n}\left[-(\lambda+\xi) q_{n}^{*}\right]+\sum_{n=0}^{\infty} p_{n}\left[\lambda \mathscr{W}_{n}^{*}(0)+\xi \mathscr{W}_{n+1}^{*}(0)\right] \\
& +\sum_{n=0}^{\infty} \int_{0}^{\infty} W_{n}(x)\left[\frac{d \mathscr{W}_{n}^{*}(x)}{d x} d x-(\lambda+\eta(x)) \mathscr{W}_{n}^{*}(x)+\lambda \mathscr{W}_{n+1}^{*}(x)+\eta(x) q_{n}^{*}\right] d x \\
= & \left\langle(p, W), \mathscr{A}^{*}\left(q^{*}, \mathscr{W}^{*}\right)\right\rangle .
\end{aligned}
$$

From the definition of adjoint operator, we conclude the desired conclusion immediately.

From Theorem 2.3, Lemma 3.1 and Arendt and Batty [18], we know that 0 is an eigenvalue of $\mathscr{A}^{*}$. Furthermore, we deduce the following result.

Lemma 3.7. If $\lambda\left(1+\frac{\lambda}{\xi}\right) \int_{0}^{\infty} x \eta(x) e^{-\int_{0}^{x} \eta_{1}(\tau) d \tau} d x<1$, then 0 is an eigenvalue of $\mathscr{A}^{*}$ with geometric multiplicity one.

Proof. We consider the equation $(\mathscr{A})^{*}\left(q^{*}, \mathscr{W}^{*}\right)=0$, which is equivalent to

$$
\begin{aligned}
& -\lambda q_{0}^{*}+\lambda \mathscr{W}_{0}^{*}(0)=0, \\
& -(\lambda+\xi) q_{n}^{*}+\xi \mathscr{W}_{n-1}^{*}(0)+\lambda \mathscr{W}_{n}^{*}(0)=0, \quad n \geq 1, \\
& \frac{d \mathscr{W}_{n}^{*}(x)}{d x}-(\lambda+\eta(x)) \mathscr{W}_{n}^{*}(x)+\lambda \mathscr{W}_{n+1}^{*}(x)+\eta(x) q_{n}^{*}=0, \quad n \geq 1
\end{aligned}
$$

It is easy to see that

$$
\left(q^{*}, \mathscr{W}^{*}\right)=\left(\left(\begin{array}{c}
\alpha \\
\alpha \\
\vdots
\end{array}\right),\left(\begin{array}{c}
\alpha \\
\alpha \\
\vdots
\end{array}\right)\right) \in D\left(A^{*}\right)
$$


is a solution of (3.42). In addition, (3.42) is equivalent to

$$
\begin{aligned}
q_{0}^{*} & =\mathscr{W}_{0}^{*}(0), \\
q_{n}^{*} & =\frac{\xi}{\lambda+\xi} \mathscr{W}_{n-1}^{*}(0)+\frac{\lambda}{\lambda+\xi} \mathscr{W}_{n}^{*}(0), \quad n \geq 1, \\
\mathscr{W}_{n+1}^{*}(x) & =\frac{1}{\lambda}\left\{-\frac{d \mathscr{W}_{n}^{*}(x)}{d x}+(\lambda+\eta(x)) \mathscr{W}_{n}^{*}(x)-\eta(x) q_{n}^{*}\right\}, \quad n \geq 1,
\end{aligned}
$$

which shows that we can determine each $q_{n}^{*}$ and $\mathscr{W}_{n}^{*}(x)$ for all $n \geq 0$ if $\mathscr{W}_{0}^{*}(x)$ is given. That is, geometric multiplicity of zero is one.

Combining Theorem 2.3, Lemma 3.1, Lemma 3.5 and Lemma 3.7 and Gupur, Li and Zhu [13, Theorem 14], we conclude the following result.

Theorem 3.8. If $0<\underline{\eta}=\inf _{x \in[0, \infty)} \eta(x) \leq \bar{\eta}=\sup _{x \in[0, \infty)} \eta(x)<\infty$, then the time-dependent solution of the system (1.7) strongly converges its steady-state solution, i.e., $\lim _{t \rightarrow \infty}\|(p, W)(\cdot, t)-\beta(p, W)(\cdot)\|=0$, here $(p, W)(x)$ is the eigenvector in Lemma 3.1 and $\beta$ is decided by the eigenvector in Lemma 3.7 and the initial value $(p, W)(0)$.

\section{CONCLUSION}

In this paper, we considered the single-line retrial queueing system with discourage repeated demands. The system is described by an infinite number of partial integro-differential equations with integral boundary conditions. We first converted the system as an abstract Cauchy problem in a proper Banach Space with corresponding operator, and then proved that the system has a unique time-dependent solution $(p, W)(x, t)=T(t)(p, W)(0), t \in[0, \infty)$, where $T(t)$ is $C_{0}-$ semigroup generated by the main operator $\mathscr{A}$. From the spectrum of the operator $\mathscr{A}$ on the right half complex plane and imaginary axis, we obtained that the time-dependent solution of the system strongly converges its steady-state solution. An interesting future research direction is to further study the spectrum of the operator on the left half complex plane so that we can describe the spectrum of the operator $\mathscr{A}$.

\section{APPENDIX}

Proof of Theorem 2.1. First, we take $\mathscr{A}=\mathscr{H}+\mathscr{U}+\mathscr{E}$, where

$$
\begin{aligned}
\mathscr{H}(p, W)= & \left(\begin{array}{ccccc}
-\lambda & 0 & 0 & 0 & \ldots \\
0 & -(\lambda+\xi) & 0 & 0 & \ldots \\
0 & 0 & -(\lambda+\xi) & 0 & \ldots \\
0 & 0 & 0 & -(\lambda+\xi) & \ldots \\
\vdots & \vdots & \vdots & \vdots & \ddots
\end{array}\right)\left(\begin{array}{c}
p_{0} \\
p_{1} \\
p_{2} \\
p_{3} \\
\vdots
\end{array}\right), \\
& \left.\left(\begin{array}{ccccc}
-\frac{d}{d x} & 0 & 0 & 0 & \ldots \\
0 & -\frac{d}{d x} & 0 & 0 & \ldots \\
0 & 0 & -\frac{d}{d x} & 0 & \ldots \\
0 & 0 & 0 & -\frac{d}{d x} & \ldots \\
\vdots & \vdots & \vdots & \vdots & \ddots
\end{array}\right)\left(\begin{array}{c}
W_{0} \\
W_{1} \\
W_{2} \\
W_{3} \\
\vdots
\end{array}\right)\right), \quad D(\mathscr{H})=D(\mathscr{A})
\end{aligned}
$$




$$
\begin{aligned}
\mathscr{U}(p, W)= & \left(\begin{array}{cccc}
0 & 0 & 0 & \ldots \\
0 & 0 & 0 & \ldots \\
0 & 0 & 0 & \ldots \\
\vdots & \vdots & \vdots & \ddots
\end{array}\right)\left(\begin{array}{c}
p_{0} \\
p_{1} \\
p_{2} \\
\vdots
\end{array}\right), \\
& \left.\left(\begin{array}{cccc}
-(\lambda+\eta(x)) & 0 & 0 & \ldots \\
0 & -(\lambda+\eta(x)) & 0 & \ldots \\
0 & 0 & -(\lambda+\eta(x)) & \ldots \\
\vdots & \vdots & \ddots
\end{array}\right)\left(\begin{array}{c}
W_{0} \\
W_{1} \\
W_{2} \\
\vdots
\end{array}\right)\right), \\
\mathscr{E}(p, W)= & \left(\left(\begin{array}{cc}
\int_{0}^{\infty} \eta(x) W_{0}(x) d x \\
\int_{0}^{\infty} \eta(x) W_{1}(x) d x \\
\int_{0}^{\infty} \eta(x) W_{2}(x) d x \\
\vdots
\end{array}\right),\left(\begin{array}{c}
0 \\
0 \\
0 \\
\vdots
\end{array}\right)\right), \quad D(\mathscr{U})=D(\mathscr{E})=X
\end{aligned}
$$

Then, we prove that $(\gamma I-\mathscr{H})^{-1}$ exists and is bounded for $\gamma>0$. For any given $(y, z) \in X$, we consider the equation $(\gamma I-\mathscr{H})(p, W)=(y, z)$. It is equivalent to the following equations

$$
\begin{aligned}
(\gamma+\lambda) p_{0} & =y_{0}, \\
(\gamma+\lambda+\xi) p_{n} & =y_{n}, \quad n \geq 1, \\
\frac{d W_{n}(x)}{d x} & =-\gamma W_{n}(x)+z_{n}(x), \quad n \geq 0, \\
W_{n}(0) & =\lambda p_{n}+\xi p_{n+1}, \quad n \geq 0 .
\end{aligned}
$$

Solving (A.1)-(A.3), we have

$$
\begin{aligned}
p_{0} & =\frac{1}{\gamma+\lambda} y_{0}, \\
p_{n} & =\frac{1}{\gamma+\lambda+\xi} y_{n}, \quad n \geq 1, \\
W_{n}(x) & =a_{n} e^{-\gamma x}+e^{-\gamma x} \int_{0}^{x} z_{n}(\tau) e^{\gamma \tau} d \tau, \quad n \geq 0 .
\end{aligned}
$$

Combining (A.5), (A.6) with (A.7), we deduce

$$
\begin{aligned}
\sum_{n=0}^{\infty}\left|a_{n}\right| & =\sum_{n=0}^{\infty}\left|W_{n}(0)\right|=\lambda \sum_{n=0}^{\infty}\left|p_{n}\right|+\xi \sum_{n=0}^{\infty}\left|p_{n+1}\right| \\
& =\frac{\lambda}{\gamma+\lambda}\left|y_{0}\right|+\frac{\lambda}{\gamma+\lambda+\xi} \sum_{n=1}^{\infty}\left|y_{n}\right|+\frac{\xi}{\gamma+\lambda+\xi} \sum_{n=1}^{\infty}\left|y_{n}\right| \\
& =\frac{\lambda}{\gamma+\lambda}\left|y_{0}\right|+\frac{\lambda+\xi}{\gamma+\lambda+\xi} \sum_{n=1}^{\infty}\left|y_{n}\right| .
\end{aligned}
$$


By using (A.8) and the Fubini Theorem, we have

$$
\begin{aligned}
\|W\| & =\sum_{n=0}^{\infty}\left\|W_{n}\right\|_{L^{1}[0, \infty)} \\
& \leq \sum_{n=0}^{\infty}\left|a_{n}\right| \int_{0}^{\infty} e^{-\gamma x} d x+\sum_{n=0}^{\infty} \int_{0}^{\infty} e^{-\gamma x} \int_{0}^{x}\left|z_{n}(\tau)\right| e^{\gamma \tau} d \tau d x \\
& \leq \frac{\lambda}{\gamma(\gamma+\lambda)}\left|y_{0}\right|+\frac{\lambda+\xi}{\gamma(\gamma+\lambda+\xi)} \sum_{n=1}^{\infty}\left|y_{n}\right|+\sum_{n=0}^{\infty} \int_{0}^{\infty}\left|z_{n}(\tau)\right| e^{\gamma \tau} \int_{\tau}^{\infty} e^{-\gamma x} d x d \tau \\
& =\frac{\lambda}{\gamma(\gamma+\lambda)}\left|y_{0}\right|+\frac{\lambda+\xi}{\gamma(\gamma+\lambda+\xi)} \sum_{n=1}^{\infty}\left|y_{n}\right|+\frac{1}{\gamma} \sum_{n=0}^{\infty}\left\|z_{n}\right\|_{L^{1}[0, \infty)} .
\end{aligned}
$$

By combining (A.5),(A.6) and (A.9), it follows (without loss of generality assume that $\gamma>0$ ) that

$$
\begin{aligned}
\|(p, W)\|= & \sum_{n=0}^{\infty}\left|p_{n}\right|+\sum_{n=0}^{\infty}\left\|W_{n}\right\|_{L^{1}[0, \infty)} \\
\leq & \frac{1}{\gamma+\lambda}\left|y_{0}\right|+\frac{1}{\gamma+\lambda+\xi} \sum_{n=1}^{\infty}\left|y_{n}\right| \\
& +\frac{\lambda}{\gamma(\gamma+\lambda)}\left|y_{0}\right|+\frac{\lambda+\xi}{\gamma(\gamma+\lambda+\xi)} \sum_{n=1}^{\infty}\left|y_{n}\right|+\frac{1}{\gamma} \sum_{n=0}^{\infty}\left\|z_{n}\right\|_{L^{1}[0, \infty)} \\
= & \frac{\gamma+\lambda}{\gamma(\gamma+\lambda)}\left|y_{0}\right|+\frac{\gamma+\lambda+\xi}{\gamma(\gamma+\lambda+\xi)} \sum_{n=1}^{\infty}\left|y_{n}\right|+\frac{1}{\gamma} \sum_{n=0}^{\infty}\left\|z_{n}\right\|_{L^{1}[0, \infty)} \\
= & \frac{1}{\gamma} \sum_{n=0}^{\infty}\left|y_{n}\right|+\frac{1}{\gamma} \sum_{n=0}^{\infty}\left\|z_{n}\right\|_{L^{1}[0, \infty)} \\
= & \frac{1}{\gamma}\|(y, z)\| .
\end{aligned}
$$

(A.10) shows that $(\gamma I-\mathscr{H})^{-1}$ exists when $\gamma>0$, and

$$
(\gamma I-\mathscr{H})^{-1}: X \rightarrow D(\mathscr{H}), \quad\left\|(\gamma I-A)^{-1}\right\|<\frac{1}{\gamma} .
$$

Next, we prove that $D(\mathscr{H})$ is dense in $X$. For $\forall(p, W) \in X$ we have

$$
\sum_{n=0}^{\infty}\left|p_{n}\right|+\sum_{n=0}^{\infty}\left\|W_{n}\right\|_{L^{1}[0, \infty)}<\infty .
$$

Hence, for any $\varepsilon>0$, there exists a finite positive integer $N$ such that

$$
\sum_{n=N}^{\infty}\left|p_{n}\right|<\varepsilon, \quad \sum_{n=N}^{\infty}\left\|W_{n}\right\|_{L^{1}[0, \infty)}<\varepsilon
$$

which shows that the set

$$
L=\left\{\begin{array}{l|l}
(p, W) & \begin{array}{l}
p=\left(p_{0}, p_{1}, p_{2}, \cdots, p_{k}, 0,0, \cdots\right) \\
W(x)=\left(W_{0}(x), W_{1}(x), \cdots, W_{k}(x), \cdots\right) \\
p_{n} \in \mathbb{R}, W_{n} \in L^{1}[0, \infty), n=0,1,2, \cdots, k \\
\mathrm{k} \text { is finite positive integer }
\end{array}
\end{array}\right\}
$$


dense in $X$. If we set

$$
V=\left\{\begin{array}{l|l}
(p, W) & \begin{array}{l}
p=\left(p_{0}, p_{1}, p_{2}, \cdots, p_{N}, 0,0, \cdots\right) \\
W(x)=\left(W_{0}(x), W_{1}(x), \cdots, W_{N}(x), \cdots\right) \\
W_{n} \in C_{0}^{\infty}[0, \infty), \text { there exists positive number } c_{i}>0 \\
\text { such that } W_{i}(x)=0, x \in\left[0, c_{i}\right], i=0,1, \cdots, N \\
\mathrm{~N} \text { is finite positive integer }
\end{array}
\end{array}\right\}
$$

then it is obvious from Adams [15] that $V$ is dense in $L$. Therefore, in order to prove that $D(\mathscr{H})$ is dense in $X$, it suffices to prove $V \subset \overline{D(\mathscr{H})}$. In fact, if $V \subset \overline{D(\mathscr{H})}$, then $X=\bar{L}=\overline{\bar{V}}=\bar{V} \subset \overline{\overline{D(\mathscr{H})}}=\overline{D(\mathscr{H})} \subset X$ implies $X=\overline{D(\mathscr{H})}$. Taking any fixed $(p, W) \in V$, we have that there are a finite positive integer $N$ and positive numbers $c_{i}>0(i=0,1, \cdots, N)$ such that

$$
\begin{aligned}
& p=\left(p_{0}, p_{1}, p_{2}, \cdots, p_{N}, 0,0, \cdots\right), \\
& W(x)=\left(W_{0}(x), W_{1}(x), \cdots, W_{N}(x), \cdots\right), \\
& W_{i}(x)=0, \text { for } x \in\left[0, c_{i}\right], i=0,1, \cdots, N .
\end{aligned}
$$

This implies $W_{i}(x)=0$, for $x \in[0, s], \quad 0<s<\min \left\{c_{0}, c_{1}, \cdots, c_{N}\right\}, \quad i=0,1, \cdots, N$. Define

$$
\begin{aligned}
f^{s}(0) & =\left(f_{0}^{s}(0), f_{1}^{s}(0), \cdots f_{N}^{s}(0), 0, \cdots\right) \\
& =\left(\lambda p_{0}+\xi p_{1}, \lambda p_{1}+\xi p_{2}, \cdots, \lambda p_{N}+\xi p_{N+1}, 0, \cdots\right), \\
f^{s}(x) & =\left(f_{0}^{s}(x), f_{1}^{s}(x), \cdots, f_{N}^{s}(0), 0, \cdots\right),
\end{aligned}
$$

where

$$
f_{i}^{s}(x)=\left\{\begin{array}{ll}
f_{i}^{s}(0)\left(1-\frac{x}{s}\right)^{2} & \text { if } x \in[0, s), \\
W_{i}(x) & \text { if } x \in[s, \infty) .
\end{array}, \quad i=0,1, \cdots, N .\right.
$$

It is easy to verify that $\left(p, f^{s}\right) \in D(A)$. Moreover

$$
\begin{aligned}
\left\|(p, W)-\left(p, f^{s}\right)\right\| & =\sum_{i=0}^{N} \int_{0}^{s}\left|W_{i}(x)-f_{i}^{s}(x)\right| d x=\sum_{i=0}^{N} \int_{0}^{s}\left|f_{i}^{s}(0)\right|\left(1-\frac{x}{s}\right)^{2} d x \\
& =\left|f_{i}^{s}(0)\right| \frac{s}{3} \rightarrow 0, \quad \text { as } \quad s \rightarrow 0 .
\end{aligned}
$$

(A.11) shows that $D(\mathscr{H})$ is dense in $V$. In other words, $D(\mathscr{H})$ is dense in $X$. From the above two steps and the Hille-Yosida theorem, we know that A generates a $C_{0}$-semigroup (see Gupur, Li and Zhu [13]).

A trivial verification shows that $\mathscr{U}$ and $\mathscr{E}$ are bounded linear operators. Hence, from the perturbation theory of $C_{0}$-semigroup, we get that $\mathscr{H}+\mathscr{U}+\mathscr{E}$ generates a $C_{0}-$ semigroup $T(t)$.

Finally, by using the idea of Gupur, Li and Zhu [13], we prove that $\mathscr{H}+\mathscr{U}+\mathscr{E}$ is a dispersive operator. For $(p, W) \in D(\mathscr{H})$, we choose

$$
\begin{aligned}
\phi_{0} & =\left(\frac{\left[p_{0}\right]^{+}}{p_{0}}, \frac{\left[p_{1}\right]^{+}}{p_{1}}, \frac{\left[p_{1}\right]^{+}}{p_{1}}, \cdots\right), \\
\phi_{1}(x) & =\left(\frac{\left[W_{0}(x)\right]^{+}}{W_{0}(x)}, \frac{\left[W_{1}(x)\right]^{+}}{W_{1}(x)}, \frac{\left[W_{1}(x)\right]^{+}}{W_{1}(x)}, \cdots\right),
\end{aligned}
$$

where

$$
\left[p_{n}\right]^{+}=\left\{\begin{array}{ll}
p_{n} & \text { if } \quad p_{n}>0 \\
0 & \text { if } \quad p_{n} \leq 0
\end{array}, \quad n \geq 0, \quad\left[W_{n}(x)\right]^{+}=\left\{\begin{array}{lll}
W_{n}(x) & \text { if } \quad W_{1}(x)>0 \\
0 & \text { if } \quad W_{n}(x) \leq 0
\end{array}, \quad n \geq 0\right.\right.
$$


If we define $\Omega=\left\{x \in[0, \infty) \mid W_{n}(x)>0\right\}$ and $\tilde{\Omega}=\left\{x \in[0, \infty) \mid W_{n}(x) \leq 0\right\}$, then

$$
\begin{aligned}
\int_{0}^{\infty} \frac{d W_{n}(x)}{d x} \frac{\left[W_{n}(x)\right]^{+}}{W_{n}(x)} d x & =\int_{\Omega} \frac{d W_{n}(x)}{d x} \frac{\left[W_{n}(x)\right]^{+}}{W_{n}(x)} d x+\int_{\tilde{\Omega}} \frac{d W_{n}(x)}{d x} \frac{\left[W_{n}(x)\right]^{+}}{W_{n}(x)} d x \\
& =\int_{\Omega} \frac{d W_{n}(x)}{d x} \frac{\left[W_{n}(x)\right]^{+}}{W_{n}(x)} d x=\int_{\Omega} \frac{d W_{n}(x)}{d x} d x \\
& =\int_{0}^{\infty} \frac{d\left[W_{n}(x)\right]^{+}}{d x} d x=-\left[W_{n}(0)\right]^{+}, \quad n \geq 0 . \\
\int_{0}^{\infty} \eta(x) W_{n}(x) d x & \leq \int_{0}^{\infty} \eta(x)\left[W_{n}(x)\right]^{+} d x, \quad n \geq 0 .
\end{aligned}
$$

Then by using the boundary conditions on $(p, W) \in D(\mathscr{H})$, we obtain that

$$
\begin{aligned}
\left\langle(\mathscr{H}+\mathscr{U}+\mathscr{E})(p, W),\left(\phi_{0}, \phi_{1}\right)\right\rangle= & \frac{\left[p_{0}\right]^{+}}{p_{0}}\left\{-\lambda p_{0}+\int_{0}^{\infty} \eta(x) W_{0}(x) d x\right\} \\
& +\sum_{n=1}^{\infty} \frac{\left[p_{n}\right]^{+}}{p_{n}}\left\{-(\lambda+\xi) p_{n}+\int_{0}^{\infty} \eta(x) W_{n}(x) d x\right\} \\
& +\sum_{n=0}^{\infty} \int_{0}^{\infty}\left\{-\frac{d W_{n}(x)}{d x}-(\lambda+\eta(x)) W_{n}(x)\right\} \frac{\left[W_{n}(x)\right]^{+}}{W_{n}(x)} d x \\
& +\sum_{n=1}^{\infty} \int_{0}^{\infty} \lambda W_{n-1}(x) \frac{\left[W_{n}(x)\right]^{+}}{W_{n}(x)} d x \\
= & -\lambda\left[p_{0}\right]^{+}-(\lambda+\xi) \sum_{n=1}^{\infty}\left[p_{n}\right]^{+}+\sum_{n=0}^{\infty} \frac{\left[p_{n}\right]^{+}}{p_{n}} \int_{0}^{\infty} \eta(x) W_{n}(x) d x \\
& -\sum_{n=0}^{\infty} \int_{0}^{\infty} \frac{d W_{n}(x)}{d x} \frac{\left[W_{n}(x)\right]^{+}}{W_{n}(x)} d x-\sum_{n=0}^{\infty} \int_{0}^{\infty}(\lambda+\eta(x))\left[W_{n}(x)\right]^{+} d x \\
& +\sum_{n=1}^{\infty} \int_{0}^{\infty} \lambda W_{n-1}(x) \frac{\left[W_{n}(x)\right]^{+}}{W_{n}(x)} d x \\
= & -\lambda \sum_{n=0}^{\infty}\left[p_{n}\right]^{+}-\xi \sum_{n=1}^{\infty}\left[p_{n}\right]^{+}+\sum_{n=0}^{\infty} \frac{\left[p_{n}\right]^{+}}{p_{n}} \int_{0}^{\infty} \eta(x) W_{n}(x) d x \\
& +\sum_{n=0}^{\infty}\left[W_{n}(0)\right]^{+}-\sum_{n=0}^{\infty} \int_{0}^{\infty}(\lambda+\eta(x))\left[W_{n}(x)\right]^{+} d x \\
& +\sum_{n=1}^{\infty} \int_{0}^{\infty} \lambda\left[W_{n-1}(x)\right]^{+} \frac{\left[W_{n}(x)\right]^{+}}{W_{n}(x)} d x \\
\leq & \sum_{n=0}^{\infty} \frac{\left[p_{n}\right]^{+}}{p_{n}} \int_{0}^{\infty} \eta(x)\left[W_{n}(x)\right]^{+} d x-\sum_{n=0}^{\infty} \int_{0}^{\infty}(\lambda+\eta(x))\left[W_{n}(x)\right]^{+} d x \\
& \left.+\lambda \sum_{n=1}^{\infty} \int_{0}^{\infty}\left[\frac{\left[W_{n}\right]^{+}}{p_{0}}-1\right) \int_{0}^{\infty} \eta(x)\right]^{+} d x \\
& \sum_{0}(x)\left[W_{n}(x)\right]^{+} d x \leq 0 . \\
& \\
&
\end{aligned}
$$

From (A.12) together with the definition of dispersive operator we know that $\mathscr{H}+\mathscr{U}+\mathscr{E}$ is a dispersive operator (see Gupur, Li and Zhu [13]). Therefor, from the first step, the second step and Phillips Theorem we know that $\mathscr{A}$ generates a positive contraction $C_{0}-$ semigroup. By uniqueness of the $C_{0}-$ semigroup it follows that this semigroup is just $T(t)$. 


\section{Funding}

This paper was supported by the National Natural Science Foundation of China under grant No.11801485.

\section{Acknowledgements}

The authors would like to express their sincere thanks to the anonymous referees and handling editor for his/her careful reading of the manuscript.

\section{REFERENCES}

[1] K. Farahmand, Single line queue with repeated demands, Queueing Syst. 6 (1990), 223-228.

[2] G.I. Falin, J.G.C. Templeton, Retrial Queues, Chapman and Hall, London, 1997.

[3] I. Atencia, I. Fortes, P. Moreno, S. Sanchez, An M/G/1 retrial queue with active breakdowns and Bernoulli schedule in the server, Int. J. Inf. Manage. Sci. 17 (2006), 1-17.

[4] J.R. Artalejo, A. Gomez-Corral, A note on the busy period of the M/G/1 queue with finite retrial group, Prob. Eng. Inform. Sci. 21 (2007),77-82.

[5] B.D. Choi, A. Melikov, A. Velibekov, A simple numerical approximation of joint probabilities of calls in service and calls in the retrial group in a picocell, Appl. Comput. Math. 7 (2008), 21-30.

[6] G. Choudhury, An M/G/1 retrial queue with an additional phase of second service and general retrial times, Int. J. Inf. Manage. Sci. 20 (2009), 1-14.

[7] J.T. Wang, L.F. Zhao, F. Zhang, Analysis of the fnite source retrial queues with server breakdowns and repairs. J. Ind. Manag. Optim. 7 (2011), 655-676.

[8] G. Gupur, Semigroup method for $M / G / 1$ retrial queue with general retrial times, Int. J. Pure Appl. Math. 18 (2005), 405-429.

[9] G. Gupur, Analysis of the M/G/1 retrial queueing model with server breakdowns, J. Pseudo-Differ. Oper. Appl. 1 (2010), 313-340.

[10] E.Kasim, G. Gupur, Other eigenvavlues of the M/M/1 operator, Acta Anal. Funct. Appl. 13(2011), 45-53.

[11] C. Gao, G.T. Zhu, Asymptotic stability of $M / G / 1$ Retrial System, Math. Pract. Theory 44 (2014), 163-169.

[12] H.O. Fattorini, The Cauchy Problem, Addison-Wesley, Massachusetts, 1983.

[13] G. Gupur, X.Z. Li, G.T. Zhu, Functional Analysis Method in Queueing Theory, Research Information Ltd., Hertfordshire, 2001.

[14] G. Greiner, Perturbing the boundary conditions of a generator, Houston J. Math. 13 (1987), 213-229.

[15] R. Adamds, Sobolev Spaces, Academic Press, New York, 1975.

[16] A. Haji, A. Radl, A semigroup approach to queueing systems, Semigroup Forum, 75 (2007), 610-624.

[17] R. Nagel, One-parameter semigroups of positive operators, Springer, Berlin, 1986.

[18] W. Arendt, C.K. Batty, Tauberian theorems and stability of one-parameter semigroups, Trans. Amer. Math. Soc. 306 (1988), 837-852. 